\title{
Robust and stochastic compliance-based topology optimization with finitely many loading scenarios
}

\author{
Mohamed Tarek • Tapabrata Ray
}

Received: date / Accepted: date

\begin{abstract}
In this paper, the problem of load uncertainty in compliance problems is addressed where the uncertainty is described in the form of a set of finitely many loading scenarios. Computationally more efficient methods are proposed to exactly evaluate and differentiate: 1) the mean compliance, or 2) any scalar-valued function of the individual load compliances such as the weighted sum of the mean and standard deviation. The computational time complexities of all the proposed algorithms are analyzed, compared with the naive approaches and then experimentally verified. Finally, a mean compliance minimization problem, a risk-averse compliance minimization problem and a maximum compliance constrained problem are solved to showcase the efficacy of the proposed algorithms. The maximum compliance constrained problem is solved using the augmented Lagrangian method and the method proposed for handling scalar-valued functions of the load compliances, where the scalar-valued function is the augmented Lagrangian function.
\end{abstract}

Keywords robust optimization · stochastic optimization · risk-averse optimization · compliance minimization - compliance constrained - SIMP . augmented Lagrangian · MMA · FEA

Mohamed Tarek

UNSW Canberra, Northcott Drive, Campbell, ACT 2600

E-mail: m.mohamed@student.adfa.edu.au

Tapabrata Ray

UNSW Canberra, Northcott Drive, Campbell, ACT 2600

E-mail: t.ray@adfa.edu.au

\section{Introduction}

1.1 Optimization under data uncertainty

Every topology optimization problem has some input data, i.e. non-decision parameters, such as the load applied or material properties. The optimal solution depends on the value of the problem's data where a change in the data can cause a significant change in the objective value or render the optimal solution obtained infeasible. Robust optimization (RO), stochastic optimization ( $\mathrm{SO})$, risk-averse optimization (RAO) and reliabilitybased design optimization (RBDO) are some of the terms used in optimization literature to describe a plethora of techniques for handling uncertainty in the data of an optimization problem.

RO describes the problem's data using an uncertainty set (Bertsimas et al., 2011). The set can be continuous, discrete or a mixed set. The main characteristic of $\mathrm{RO}$ problems is that the constraints are required to be feasible for every data point in the uncertainty set. For more on $\mathrm{RO}$, the readers are referred to Bertsimas et al. (2011) and Aharon Ben-Tal et al. (2009).

SO and RAO assume that the data follows a known probability distribution (Shapiro et al., 2009, Choi et al., 2007 ). Let $\boldsymbol{f}$ be a random load and $\boldsymbol{x}$ be the topology design variables. A probabilistic constraint can be defined as $P(g(\boldsymbol{x} ; \boldsymbol{f}) \leq 0) \geq \eta$ where $\boldsymbol{f}$ follows a known probability distribution. This constraint is often called a chance constraint or a reliability constraint in RBDO. The objective of an SO problem is typically either deterministic or some probabilistic function such as the mean of a function of the random variable, its variance, standard deviation or a weighted sum of such terms.

RAO can be considered a sub-field of SO which borrows concepts from risk analysis in mathematical eco- 
nomics to define various risk measures and tractable approximations to be used in objectives and/or constraints in SO. One such risk measure is the conditional value-at-risk (CVaR) (Shapiro et al. 2009). Other more traditional risk measures include the weighted sum of the mean and variance of a function or the weighted sum of the mean and standard deviation. For more on $\mathrm{SO}$ and RAO, the reader is referred to Shapiro et al. (2009).

RBDO and its ancestor, reliability analysis, are more commonly found in the sizing optimization literature. Classically, RBDO has been about solving optimization problems with a probabilistic constraint, called the reliability constraint, much like SO. One of the most common RBDO techniques used in topology optimization literature is the first-order reliability method (FORM). In FORM, the random variable $\boldsymbol{f}$ is assumed to be a function of a multivariate unit Gaussian random variable $\boldsymbol{u}$ relying on linearization and a Gaussian approximation of the probabilistic function's output. This approximation approach is known as the firstorder second-moment (FOSM) approach. The choice of the linearization point $\boldsymbol{u}_{0}$ affects the accuracy of FOSM, where the mean $\mathbf{0}$ is typically outperformed by the less obvious alternative known as the most probable point (MPP) $\boldsymbol{u}^{*}$. There are two ways to define the MPP point: the reliability index approach (RIA) (Yu et al. 1998, Tu et al. 1999) and the performance measure approach (PMA) (Tu et al., 1999). For more on RBDO and reliability analysis, the reader is referred to Choi et al. (2007) and Youn and Choi (2004). While classic RBDO has been about handling probabilistic reliability constraints, more recently the non-probabilistic RBDO (NRBDO) was developed, applying similar techniques as in classic RBDO but for handling set-based, nonprobabilistic uncertainty to solve RO problems (Luo et al., 2009, Kang and Luo, 2009, Guo and Lu, 2015; Zheng et al., 2018, Wang et al., 2019b a).

In topology optimization literature, the term "robust topology optimization" is often used to refer to minimizing the weighted sum of the mean, and variance or standard deviation of a function subject to probabilistic uncertainty (Dunning and Kim, 2013, Zhao and Wang, 2014b, Cuellar et al., 2018). However, this use of the term "robust optimization" is not consistent with the standard definition of RO in optimization theory literature, e.g. Ben-Tal et al. Aharon Ben-Tal et al. (2009). The more compliant term is stochastic topology optimization or risk-averse topology optimization.

The vast majority of works in literature on handling load uncertainty assume the load follows a probability distribution or lies in a continuous uncertainty set. In practice if a number of loading scenarios are known from sensor data, there is no way to use this data to perform risk-averse or robust compliance-based topology optimization. In this paper, computationally efficient approaches are proposed to compute and differentiate the mean compliance, its standard deviation and any scalar valued function of individual load compliances where each compliance is computed from a particular loading scenario. These approaches can then be used in risk-averse compliance minimization as well as handling robust compliance constraints where the uncertainty is described in the form of a set of finite loading scenarios.

\subsection{Solid isotropic material with penalization}

In this paper, the solid isotropic material with penalization (SIMP) method (Bendsoe, 1989, Sigmund, 2001 Rojas-Labanda and Stolpe, 2015) is used to solve the topology optimization problems. Let $0 \leq x_{e} \leq 1$ be the decision variable associated with element $e$ in the ground mesh and $\boldsymbol{x}$ be the vector of such decision variables. Let $\rho_{e}$ be the pseudo-density of element $e$, and $\boldsymbol{\rho}(\boldsymbol{x})$ be the vector of such variables after sequentially applying to $\boldsymbol{x}$ :

1. A chequerboard density filter typically of the form $f_{1}(\boldsymbol{x})=\boldsymbol{A} \boldsymbol{x}$ for some constant matrix $\boldsymbol{A}$ (Bendsoe and Sigmund, 2004),

2. An interpolation of the form $f_{2}(y)=\left(1-x_{m i n}\right) y+$ $x_{\text {min }}$ applied element-wise for some small $x_{\text {min }}>0$ such as 0.001 ,

3. A penalty such as the power penalty $f_{3}(z)=z^{p}$ applied element-wise for some penalty value $p$, and

4. A projection method such as the regularized Heaviside projection (Guest et al. 2004) applied elementwise.

The compliance of the discretized design is defined as: $C=\boldsymbol{u}^{T} \boldsymbol{K} \boldsymbol{u}=\boldsymbol{f}^{T} \boldsymbol{K}^{-1} \boldsymbol{f}$ where $\boldsymbol{K}$ is the stiffness matrix, $\boldsymbol{f}$ is the load vector, and $\boldsymbol{u}=\boldsymbol{K}^{-1} \boldsymbol{f}$ is the displacement vector. The relationship between the global and element stiffness matrices is given by $\boldsymbol{K}=\sum_{e} \rho_{e} \boldsymbol{K}_{e}$ where $\boldsymbol{K}_{e}$ is the hyper-sparse element stiffness matrix of element $e$ with the same size as $\boldsymbol{K}$.

\subsection{Mean compliance minimization}

A number of works in literature tackled the problem of load uncertainty in compliance minimization problems. Table 1 summarizes the literature on mean compliance minimization subject to different types of uncertainty. Nearly all the algorithms in literature assume the load follows a known probability distribution. Of all the works reviewed, only one work (Zhang et al. (2017)) 
dealt with data-driven design with no distribution assumed. Zhang et. al. assumed the load can be any one of a finite number of loading scenarios where the loading scenarios can be collected from data. The algorithm proposed by Zhang et. al. can be trivially modified to handle weighted mean compliance which can be used in cases where the number of random variables are many following the approach by Zhao and Wang (2014b) or when the number of terms of the Karhunen-Loeve (KL) expansion is high. However, the main limitation of this approach is that it can only be used to minimize the mean compliance which is not risk-averse since at the optimal solution, the compliance can still be very high for some probable load scenarios even if the mean compliance is minimized. This is one of the problems addressed in this work.

\subsection{Risk-averse compliance minimization}

Some authors studied risk-averse compliance minimization by considering the weighted sum of the mean and variance, the weighted sum of the mean and standard deviation, as well as other risk measures. Table 2 summarizes the literature on risk-averse compliance minimization. All the works reviewed assumed the load to follow a known distribution or random field with a known covariance kernel. A number of works used the K-L expansion to handle the uncertainty when described using a random field. In all the works which use K-L expansion and sampling-based uncertainty propagation, the number of linear system solves can be made independent from the number of sampling points given the linearity assumption of the displacement as a function of the load exploited by Zhao and Wang (2014a) in their derivation, even though in some of the works this property was not exploited. The number of linear system solves can therefore be assumed to be equal to the number of terms in the K-L expansion only, not the sampling points. There are no reports to the authors' knowledge on handle risk-averse compliance minimization when there is a finite set of loading scenarios, i.e. distribution-free. This work addresses this problem.

1.5 Probabilistic constraints and reliability-based topology optimization

RBDO offers a number of techniques for efficient, approximate uncertainty propagation which can be used for handling probabilistic constraints involving compliance or otherwise. Most papers handling probabilistic constraints used methods from RBDO. Table 3 summarizes the literature on probabilistic constraint handling methods that can be used in compliance-based problems.

\subsection{Maximum compliance constraint}

A number of works studied maximum compliance minimization and maximum compliance constrained problems under uncertain loading conditions. In these papers, the load was assumed to lie in a continuous uncertainty set, where no probability distribution is assumed. Therefore, they fall under the category of RO. A number of papers were also published on non-probabilistic reliability-based topology optimization (NRBTO) where new reliability indexes and performance measures are defined for various types of continuous uncertainty sets. While some of these works did not solve problems with maximum compliance constraints, the same techniques can be applied to handle maximum compliance constraints. Table 4 summarizes the literature on maximum compliance-constrained optimization including algorithms that can in theory be used to solve this class of problems. None of the reviewed papers handled the case of a finite number of loading scenarios instead of a continuous uncertainty set. This work addresses this issue.

\subsection{Paper organization}

The rest of this paper is organized as follows. The proposed approaches for handling load uncertainty in continuum compliance problems in the form of a large, finite number of loading scenarios are detailed in sections 2, 3 and 4. The experiments used and the implementations are then described in section 5 . Finally, the results are presented and discussed in section 6 before concluding in section 7 .

\section{Compliance sample mean and its gradient}

\subsection{Naive approach}

The compliance sample mean for a finite number $L$ of loading scenarios is $\mu_{C}=\frac{1}{L} \sum_{i=1}^{L} \boldsymbol{f}_{i}^{T} \boldsymbol{K}^{-1} \boldsymbol{f}_{i}$ where $\boldsymbol{f}_{i}$ is the $i^{\text {th }}$ load scenario, $\boldsymbol{K}$ is the stiffness matrix of the design and $\boldsymbol{F}$ is the matrix whose columns are the individual loading scenarios $\boldsymbol{f}_{i}$. The direct naive approach is to solve for $\boldsymbol{K}^{-1} \boldsymbol{f}_{i}$ for all $i$ and calculate the mean compliance using the above formula. This method is not efficient since it requires $L$ linear system solves plus some additional work to compute the mean with a time complexity of $O\left(L \times n_{d o f s}\right)$, where $n_{d o f s}$ is the 
Table 1: Summary of literature on mean compliance minimization.

\begin{tabular}{|c|c|c|}
\hline Paper & Uncertainty type & Summary \\
\hline \begin{tabular}{|l|} 
Guest $\quad$ and \\
Igusa 2008$)$
\end{tabular} & $\begin{array}{l}\text { Load components and load } \\
\text { locations as distributions } \\
\text { with arbitrary covariance }\end{array}$ & $\begin{array}{l}\text { General scheme for handling uncertain loads and load location. Derived } \\
\text { an approximately equivalent load distribution to result in the same com- } \\
\text { pliance as the random node location. }\end{array}$ \\
\hline $\begin{array}{l}\text { Dunning et al. } \\
(2011)\end{array}$ & $\begin{array}{l}\text { Concentrated load magni- } \\
\text { tude and direction as distri- } \\
\text { butions }\end{array}$ & $\begin{array}{l}\text { Derived efficient formulations for the mean compliance, where the number } \\
\text { of linear systems to be solved scales linearly with the number of inde- } \\
\text { pendent random variables. Assumes that the distribution of the forces' } \\
\text { magnitudes and rotations are known and independent. }\end{array}$ \\
\hline \begin{tabular}{|l|l|} 
Zhao $\quad$ and \\
Wang \\
$2014 \mathrm{~b})$
\end{tabular} & $\begin{array}{l}\text { Concentrated load magni- } \\
\text { tude and direction and dis- } \\
\text { tributed load as distributions } \\
\text { or random fields }\end{array}$ & $\begin{array}{l}\text { Requires fewer linear system solves per independent random variable } \\
\text { compared to the approach by Dunning et al. (2011). Demonstrated how } \\
\text { the same approach can handle distributed load uncertainty, modeled as } \\
\text { a stochastic field, using K-L expansion. }\end{array}$ \\
\hline $\begin{array}{lll}\text { Zhang } & \text { et } & \text { al. } \\
(2017) & \end{array}$ & Finite set of load scenarios & $\begin{array}{l}\text { Proposed an efficient way to compute the mean compliance. Developed a } \\
\text { randomized algorithm inspired by Hutchinson's trace estimator Hutchin- } \\
\text { son (1990) to minimize the mean compliance leading to significant com- } \\
\text { putational savings compared to the naive approach. }\end{array}$ \\
\hline $\begin{array}{l}\text { Liu and Wen } \\
2018)\end{array}$ & Fuzzy load & Modeled the load uncertainty using the fuzzy set theoretic cloud model. \\
\hline
\end{tabular}

Table 2: Summary of literature on risk-averse compliance minimization.

\begin{tabular}{|c|c|c|}
\hline Paper & $\begin{array}{l}\text { Uncertainty } \\
\text { type }\end{array}$ & Summary \\
\hline \begin{tabular}{|l|} 
Dunning and \\
Kim $(2013)$ \\
\end{tabular} & $\begin{array}{l}\text { Load mag- } \\
\text { nitudes as } \\
\text { independent } \\
\text { distributions }\end{array}$ & $\begin{array}{l}\text { Derived an efficient formulation for the variance of the compliance. This was used to } \\
\text { minimize a weighted sum of the mean and variance of the compliance. }\end{array}$ \\
\hline \begin{tabular}{|l} 
Zhao and \\
Wang \\
\end{tabular} & $\begin{array}{l}\text { Load as a ran- } \\
\text { dom field }\end{array}$ & $\begin{array}{l}\text { Used K-L expansion to quantify and describe the randomness using a few random vari- } \\
\text { ables, and used Monte Carlo simulation to calculate the fourth moment of those random } \\
\text { variables which is required for the efficient computation of the standard deviation of } \\
\text { the compliance. Minimized the weighted sum of the mean and standard deviation of } \\
\text { the compliance. }\end{array}$ \\
\hline \begin{tabular}{|lll} 
Chen et al. \\
$2010)$ &
\end{tabular} & $\begin{array}{l}\text { Load and ma- } \\
\text { terial proper- } \\
\text { ties as random } \\
\text { fields }\end{array}$ & $\begin{array}{l}\text { Used K-L expansion to reduce the random field's dimensionality followed by Gaussian } \\
\text { quadrature sampling to generate a representative set of scenarios to formulate the mean } \\
\text { and variance of the compliance. Used the level-set method to minimize the weighted } \\
\text { sum of the mean compliance and its variance. }\end{array}$ \\
\hline \begin{tabular}{|l|} 
Martínez- \\
Frutos and \\
Herrero-Pérez \\
$2016)$ \\
\end{tabular} & $\begin{array}{l}\text { Load as a ran- } \\
\text { dom field }\end{array}$ & $\begin{array}{l}\text { Used K-L expansion to reduce the random field's dimensionality followed by sparse grid } \\
\text { sampling to generate a representative set of scenarios to formulate the mean and vari- } \\
\text { ance of the compliance. Developed a multi-GPU density-based topology optimization } \\
\text { framework for the large-scale minimization of the weighted sum of the mean compliance } \\
\text { and its variance. }\end{array}$ \\
\hline \begin{tabular}{|l|} 
Cuellar et al. \\
$2018)$
\end{tabular} & $\begin{array}{l}\text { Load and ma- } \\
\text { terial proper- } \\
\text { ties as random } \\
\text { fields }\end{array}$ & $\begin{array}{l}\text { Used K-L expansion for uncertainty quantification and Gaussian quadrature for sam- } \\
\text { pling, and combined them with the non-intrusive polynomial chaos expansion (PCE) } \\
\text { method to provide more accurate estimators for the mean and standard deviation of } \\
\text { the compliance and their gradients. }\end{array}$ \\
\hline \begin{tabular}{|l|} 
Martínez- \\
Frutos et al. \\
2018 \\
\end{tabular} & $\begin{array}{l}\text { Load and ma- } \\
\text { terial proper- } \\
\text { ties as random } \\
\text { fields }\end{array}$ & $\begin{array}{l}\text { Used K-L expansion and the non-intrusive PCE with sparse grid sampling for the quan- } \\
\text { tification and propagation of the uncertainty in the load and material properties. Min- } \\
\text { imized a different compliance risk measure called the excess probability, which is the } \\
\text { probability that the compliance exceeds a certain threshold value. }\end{array}$ \\
\hline \begin{tabular}{|l|l|}
\multicolumn{2}{|c|}{ Garcia-Lopez } \\
et al. (2013)
\end{tabular} & $\begin{array}{l}\text { Load as a dis- } \\
\text { tribution }\end{array}$ & $\begin{array}{l}\text { Used multi-objective evolutionary optimization to optimize the mean and variance of } \\
\text { the compliance and obtain the Pareto front of the two objectives. Used a sampling } \\
\text { method for uncertainty propagation inspired from Taguchi's method for the design of } \\
\text { experiments. In this case, the number of linear system solves is equal to the number of } \\
\text { sampling points. That beside the use of an evolutionary algorithm which requires many } \\
\text { evaluations of the mean and variance of the compliance make the computational cost } \\
\text { of this approach extremely high even for medium-sized problems. }\end{array}$ \\
\hline \begin{tabular}{|l|}
\multicolumn{2}{l}{ Kriegesmann } \\
and Lüdeker \\
2019
\end{tabular} & $\begin{array}{l}\text { Load as a dis- } \\
\text { tribution }\end{array}$ & $\begin{array}{l}\text { Used FOSM instead of sampling to efficiently propagate the uncertainty estimating the } \\
\text { mean and standard deviation of the compliance and their gradients from the means and } \\
\text { standard deviations of the loads. A weighted sum of the mean and standard deviation } \\
\text { of the compliance was then minimized. This approach assumes that the compliance is } \\
\text { a linear function of the random load centered at the MPP load, an assumption which } \\
\text { leads to a prediction error in the mean and standard deviation of the compliance. }\end{array}$ \\
\hline
\end{tabular}


Table 3: Summary of literature on probabilistically constrained (or reliability constrained) compliance-based optimization.

\begin{tabular}{|c|c|}
\hline Paper & ummary \\
\hline $\begin{array}{l}\text { Keshavarzzadeh } \\
\text { et al. (2017) }\end{array}$ & $\begin{array}{l}\text { Solved the problem of volume minimization subject to a probabilistic compliance constraint. In one } \\
\text { case, the authors constrained the mean compliance plus a multiple of its standard deviation which is } \\
\text { equivalent to a reliability constraint assuming the compliance is normally distributed. In another, a } \\
\text { reliability constraint was used such that the probability that the compliance exceeds a threshold value } \\
\text { is constrained. Keshavarzzadeh et al. used the non-intrusive PCE and regularized Heaviside function } \\
\text { to approximate the compliance reliability constraint and its gradient. PCE was also used to estimate } \\
\text { the mean and standard deviation of the compliance and their gradients. }\end{array}$ \\
\hline Kharmanda & \multirow[b]{2}{*}{$\begin{array}{l}\text { Proposed the use of RBDO for topology optimization, also known as reliability-based topology op- } \\
\text { timization (RBTO), to handle probabilistic constraints due to random loads, geometry and material } \\
\text { properties. }\end{array}$} \\
\hline \begin{tabular}{|l|l|} 
and & Olhoff \\
$(2002) ;$ & Khar- \\
manda & et al. \\
$(2004)$ & \\
\end{tabular} & \\
\hline Jung and Cho & $\begin{array}{l}\text { Used FORM's PMA with SIMP t } \\
\text { for geometrically nonlinear struct }\end{array}$ \\
\hline Kim et al. & $\begin{array}{l}\text { Used FORM's RIA and PMA with SIMP to solve volume minimization problems with reliability } \\
\text { constraints on the displacement and natural frequency of the structure under loading, material and } \\
\text { geometry uncertainties. }\end{array}$ \\
\hline$\frac{\text { Kim et al. }}{(2007,2008)}$ & 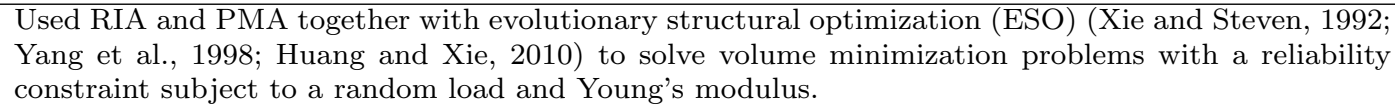 \\
\hline$\frac{\text { Ouyang et al. }}{(2008)}$ & $\begin{array}{l}\text { Used FORM's RIA with the level-set method to solve a compliance minimization problem with a } \\
\text { reliability constraint subject to uncertainty in the load and geometry of the ground mesh. }\end{array}$ \\
\hline Silva et al. & $\begin{array}{l}\text { proposed the use of an efficiently obtainable approximate MPP to avoid the need for solving the } \\
\text { reliability or inverse reliability problems in every design iteration of RIA or PMA, respectively. }\end{array}$ \\
\hline $\begin{array}{l}\text { Silva et al. } \\
(2010) ; \\
\text { Nguyen et al. } \\
(2011)\end{array}$ & $\begin{array}{l}\text { Considered system reliability-based topology optimization, where an aggregated system failure prob- } \\
\text { ability is considered instead of component failure probabilities and component limit state functions. }\end{array}$ \\
\hline Zhao et al. & $\begin{array}{l}\text { Presented a comparison of a number of RBTO approaches to solve a few topology optimization } \\
\text { problems including one with a compliance reliability constraint under stochastic load and Young's } \\
\text { modulus. }\end{array}$ \\
\hline \begin{tabular}{|l} 
Jalalpour and \\
Tootkaboni \\
$2016)$ \\
\end{tabular} & $\begin{array}{l}\text { Developed a bi-directional ESO (BESO) Xie and Steven 1992, Yang et al. } 1998 \text { Huang and Xie } \\
\text { 2010) algorithm for handling reliability constraints with displacement limit state functions and a finite } \\
\text { number of probable loading scenarios in linearly elastic structures. }\end{array}$ \\
\hline Yin et al. & ing fuzzy set theory to describe the uncertainty. \\
\hline
\end{tabular}

number of degrees of freedom in the design. When $\boldsymbol{F}$ is sparse with only a few $n_{\text {loaded }}$ degrees of freedom that are loaded, the complexity of the remaining work to compute the mean compliance $\frac{1}{L} \sum_{i=1}^{L} \boldsymbol{f}_{i}^{T} \boldsymbol{u}_{i}$ becomes $O\left(L \times n_{\text {loaded }}\right)$. Even though the factorization of $\boldsymbol{K}^{-1}$ can be reused to solve for the $L$ linear systems, if $L$ is close to $n_{\text {dofs }}$, the complexity of solving for so many linear systems will be similar to that of the factorization, thus significantly adding to the running time. When using an iterative algorithm to solve for $\boldsymbol{K}^{-1} \boldsymbol{f}_{i}$, a good, but expensively formed, preconditioner such as the algebraic multi-grid preconditioner can be similarly reused. In general, significantly reducing the number of linear systems to solve is advantageous in practice even if, as theory may show, the running time is dominated by the initial linear system solve.

Let the Jacobian of $\boldsymbol{\rho}(\boldsymbol{x})$ be $\nabla_{\boldsymbol{x}} \boldsymbol{\rho}(\boldsymbol{x})$. Let $\boldsymbol{u}_{i}$ be the displacement response due to load $\boldsymbol{f}_{i}$ and $C_{i}$ be the compliance $\boldsymbol{f}_{i}^{T} \boldsymbol{u}_{i}$. The stiffness matrix $\boldsymbol{K}$ is typi- cally defined as: $\boldsymbol{K}=\sum_{e} \rho_{e} \boldsymbol{K}_{e}$. The partial derivative of the compliance $C_{i}$ with respect to $\rho_{e}$ is given by $\frac{\partial C_{i}}{\partial \rho_{e}}=-\boldsymbol{u}_{i}^{T} \boldsymbol{K}_{e} \boldsymbol{u}_{i}$. The gradient of $C_{i}$ with respect to the decision vector $\boldsymbol{x}$ is therefore given by: $\nabla_{\boldsymbol{x}} C_{i}(\boldsymbol{x})=$ $\nabla_{\boldsymbol{x}} \boldsymbol{\rho}(\boldsymbol{x})^{T} \nabla_{\boldsymbol{\rho}} C_{i}(\boldsymbol{\rho}(\boldsymbol{x}))$ where $\nabla_{\boldsymbol{\rho}} C_{i}(\boldsymbol{\rho}(\boldsymbol{x}))$ is the gradient of $C_{i}$ with respect to $\boldsymbol{\rho}$ at $\boldsymbol{\rho}(\boldsymbol{x})$. The gradient of the mean compliance $\mu_{C}$ is therefore given by $\nabla_{\boldsymbol{x}} \mu_{C}(\boldsymbol{x})=$ $\frac{1}{L} \sum_{i=1}^{L} \nabla_{\boldsymbol{x}} \boldsymbol{\rho}(\boldsymbol{x})^{T} \nabla_{\boldsymbol{\rho}} C_{i}(\boldsymbol{\rho}(\boldsymbol{x}))$. The additional complexity of computing the mean compliance and its gradient with respect to $\boldsymbol{\rho}$ is $O\left(n_{E} \times L\right)$. Note that the Jacobian of $\boldsymbol{\rho}(\boldsymbol{x})$ does not need to be formed explicitly to compute the gradient above, so long as there is a way to pre-multiply the Jacobian's transpose by a vector. The problem with the naive approach is it requires many linear system solves and so doesn't scale well to many loading scenarios. 
Table 4: Summary of literature on maximum compliance constrained optimization.

\begin{tabular}{|c|c|c|}
\hline Paper & Uncertainty type & Summary \\
\hline \begin{tabular}{|l|} 
Brittain \\
et al. \\
$(2012)$
\end{tabular} & $\begin{array}{l}\text { Load vector with a } \\
\text { fixed norm and arbi- } \\
\text { trary direction }\end{array}$ & $\begin{array}{l}\text { Used a bi-level min-max optimization approach minimizing the objective with re- } \\
\text { spect to the topology variables in the upper level problem, and maximizing with } \\
\text { respect to the load in the lower level problem. However, an efficient algorithm was } \\
\text { derived for the lower level maximization problem based on the KKT optimality } \\
\text { conditions for the objective and the load's fixed-norm constraint. }\end{array}$ \\
\hline \begin{tabular}{l|l|} 
Holmber \\
et al. \\
$(2015)$ \\
\end{tabular} & $\begin{array}{l}\text { Load vector in a } \\
\text { hyper-ellipsoid }\end{array}$ & $\begin{array}{l}\text { Proposed a nonlinear semi-definite formulation to solve the set-maximum compli- } \\
\text { ance minimization problem. }\end{array}$ \\
\hline \begin{tabular}{l|} 
Thore \\
et al. \\
$(2017)$ \\
\end{tabular} & $\begin{array}{l}\text { Load vector in a } \\
\text { hyper-ellipsoid }\end{array}$ & $\begin{array}{l}\text { Generalized the approach from Holmberg et al. }(2015) \text { to handle maximum com- } \\
\text { pliance and maximum stress constraints under the same assumption on the load } \\
\text { vector. }\end{array}$ \\
\hline \begin{tabular}{|l} 
Liu \\
and \\
Gea \\
2018 \\
\end{tabular} & $\begin{array}{l}\text { Multiple independent } \\
\text { loads each in a hyper- } \\
\text { ellipsoid }\end{array}$ & $\begin{array}{l}\text { Proposed a bi-level formulation. Developed an efficient lower level algorithm by } \\
\text { solving the Wolfe dual problem. The Wolfe dual problem of the lower level problem } \\
\text { is a maximum generalized eigenvalue minimization problem which was solved using } \\
\text { an iterative procedure. The multi-ellipsoidal uncertainty set generalizes the interval } \\
\text { as well as the spherical uncertainty sets. }\end{array}$ \\
\hline \begin{tabular}{|l|} 
Luo \\
et al. \\
$(2009)$ \\
\end{tabular} & $\begin{array}{l}\text { Generic uncertain } \\
\text { variables in a multi- } \\
\text { ellipsoid set }\end{array}$ & $\begin{array}{l}\text { Proposed an NRBDO reliability index and performance measure for handling non- } \\
\text { probabilistic uncertainty. }\end{array}$ \\
\hline \begin{tabular}{|l|} 
Wang \\
et al. \\
$(2018)$ \\
\end{tabular} & $\begin{array}{l}\text { Generic uncertain } \\
\text { variables in an ellip- } \\
\text { soid }\end{array}$ & $\begin{array}{l}\text { Proposed another NRBDO reliability index for handling non-probabilistic uncer- } \\
\text { tainty. }\end{array}$ \\
\hline \begin{tabular}{|l|} 
Wang \\
et al. \\
2017 \\
$2019 \mathrm{~b}$ \\
\end{tabular} & $\begin{array}{l}\text { Generic uncertain } \\
\text { variables in an inter- } \\
\text { val }\end{array}$ & Proposed an NRBDO reliability index using interval arithmetic. \\
\hline \begin{tabular}{|l|} 
Zheng \\
et al. \\
$2018)$
\end{tabular} & $\begin{array}{l}\text { Generic uncer- } \\
\text { tain variables in } \\
\text { multidimensional } \\
\text { parallelepiped convex } \\
\text { sets }\end{array}$ & Proposed an NRBDO reliability index and performance function. \\
\hline \begin{tabular}{|l|}
\multicolumn{2}{|l|}{ Wang } \\
et al. \\
$2019 \mathrm{a}$
\end{tabular} & $\begin{array}{l}\text { Generic uncertain } \\
\text { variables in a mixed } \\
\text { interval and ellip- } \\
\text { soidal set }\end{array}$ & Proposed an NRBDO reliability index. \\
\hline
\end{tabular}

\subsection{Singular value decomposition}

Less naively, one can first attempt to find the singular value decomposition (SVD) of $\boldsymbol{F}$. Let the compact SVD of the matrix $\boldsymbol{F}$ be $\boldsymbol{F}=\boldsymbol{U} \boldsymbol{S} \boldsymbol{V}^{T}$, where the number of non-zero singular values is $n_{s}, \boldsymbol{S}$ is the diagonal matrix of singular values, $\boldsymbol{U}$ is a $n_{\text {dofs }} \times n_{s}$ matrix with orthonormal columns, and $\boldsymbol{V}$ is $L \times n_{s}$ matrix with orthonormal columns. Given the SVD, the mean compliance can be written as: $\mu_{C}=\frac{1}{L} \sum_{i=1}^{L} \boldsymbol{f}_{i}^{T} \boldsymbol{K}^{-1} \boldsymbol{f}_{i}=$ $\frac{1}{L} \operatorname{tr}\left(\boldsymbol{F}^{T} \boldsymbol{K}^{-1} \boldsymbol{F}\right)$. This can be further simplified:

$$
\begin{aligned}
\frac{1}{L} \operatorname{tr}\left(\boldsymbol{F}^{T} \boldsymbol{K}^{-1} \boldsymbol{F}\right) & =\frac{1}{L} \operatorname{tr}\left(\boldsymbol{V} \boldsymbol{S} \boldsymbol{U}^{T} \boldsymbol{K}^{-1} \boldsymbol{U} \boldsymbol{S} \boldsymbol{V}^{T}\right) \\
& =\frac{1}{L} \operatorname{tr}\left(\boldsymbol{S} \boldsymbol{U}^{T} \boldsymbol{K}^{-1} \boldsymbol{U} \boldsymbol{S}\right) \\
& =\frac{1}{L} \sum_{i=1}^{n_{s}} \boldsymbol{S}[i, i]^{2} \times \boldsymbol{U}[:, i]^{T} \boldsymbol{K}^{-1} \boldsymbol{U}[:, i]
\end{aligned}
$$

This method requires only $n_{s}$ linear system solves and an SVD. $n_{s}$ will be small if the loads in $\boldsymbol{F}$ are highly correlated or if only a few degrees of freedom are loaded, i.e. the loads are sparse. Let $n_{\text {loaded }}$ be the few loaded degrees of freedom. It is possible to prove in this case that the number of singular values $n_{s} \leq n_{\text {loaded }}$. The computational time complexity of computing the SVD of $\boldsymbol{F}$ in the dense case is $O\left(\min \left(L, n_{\text {dofs }}\right)^{2} \max \left(L, n_{\text {dofs }}\right)\right)$, while in the sparse case it is only $O\left(n_{\text {loaded }}^{2} L\right)$. If $n_{\text {loaded }}$ is a small constant, finding the SVD will be very efficient. Additionally, when only $n_{\text {loaded }}$ degrees of freedom are loaded in $\boldsymbol{F}$, only the same degrees of freedom will be non-zero in $\boldsymbol{U}$, therefore $\boldsymbol{U}$ will also be sparse. Other than the complexity of SVD, the additional work to compute the mean compliance has a computational time complexity of $O\left(n_{s} \times n_{\text {dofs }}\right)$ when $\boldsymbol{F}$ (and $\left.\boldsymbol{U}\right)$ are dense, and $O\left(n_{s} \times n_{\text {loaded }}\right)$ when $\boldsymbol{F}$ (and $\left.\boldsymbol{U}\right)$ are sparse.

Given the efficient formula for the mean compliance and using the derivative rule of the inverse quadratic from the appendix, the partial $\frac{\partial \mu_{C}}{\partial \rho_{e}}$ is given by: $-\frac{1}{L} \sum_{i=1}^{n_{s}} \boldsymbol{S}[i, i]^{2}\left(\boldsymbol{K}^{-1} \boldsymbol{U}\right)[:, i]^{T} \boldsymbol{K}_{e}\left(\boldsymbol{K}^{-1} \boldsymbol{U}\right)[:, i]$. The time 
complexity of computing this assuming we already computed $\boldsymbol{K}^{-1} \boldsymbol{U}$ is $O\left(n_{s} \times n_{E}\right)$.

\section{Scalar-valued function of load compliances and its gradient}

In this section, the above approach for computing the sample mean compliance will be generalized to handle the sample variance and standard deviations. The sample variance of the compliance $C$ is given by $\sigma_{C}^{2}=$ $\frac{1}{L-1} \sum_{i=1}^{L}\left(C_{i}-\mu_{C}\right)^{2}$. The sample standard deviation $\sigma_{C}$ is the square root of the variance. Let $\boldsymbol{C}$ be the vector of compliances $C_{i}$, one for each load scenario. In vector form, $\sigma_{C}^{2}=\frac{1}{L-1}\left(\boldsymbol{C}-\mu_{C} \mathbf{1}\right)^{T}\left(\boldsymbol{C}-\mu_{C} \mathbf{1}\right) . \boldsymbol{C}=$ $\operatorname{diag}(\boldsymbol{A})$ is the diagonal of the matrix $\boldsymbol{A}=\boldsymbol{F}^{T} \boldsymbol{K}^{-1} \boldsymbol{F}$.

\subsection{Naive approach}

If one can compute the vector of load compliances $\boldsymbol{C}$, computing the variance and standard deviation is trivial. This approach requires $L$ linear system solves which can be computationally prohibitive if $L$ is large. Other than the linear system solves, the remaining work of computing $C_{i}=\boldsymbol{f}_{i}^{T} \boldsymbol{u}_{i}$ for all $i$ has a complexity of $O\left(L \times n_{\text {dofs }}\right)$ when $\boldsymbol{F}$ is dense and $O\left(L \times n_{\text {loaded }}\right)$ when $\boldsymbol{F}$ is sparse with only $n_{\text {loaded }}$ loaded degrees of freedom. In order to compute the vector $\boldsymbol{C}$ exactly, one needs to compute $\boldsymbol{u}_{i}=\boldsymbol{K}^{-1} \boldsymbol{f}_{i}$ for all $i$. These can further be used to compute the gradients of the load compliances $C_{i}$ which can be combined to form the Jacobian $\nabla_{\rho} \boldsymbol{C}$. Assuming $\boldsymbol{u}_{i}$ is cached for all $i$, the time complexity of computing the Jacobian using $\frac{\partial C_{i}}{\partial \rho_{e}}=-\boldsymbol{u}_{i}^{T} \boldsymbol{K}_{e} \boldsymbol{u}_{i}$ is $O\left(n_{E} \times L\right)$.

However, when interested in the gradient of a scalarvalued function $f$ of $\boldsymbol{C}$, there is no need to form the full Jacobian $\nabla_{\boldsymbol{x}} \boldsymbol{C}(\boldsymbol{x})$. It suffices to define an operator to pre-multiply an arbitrary vector $\boldsymbol{w}$ by $\nabla_{\boldsymbol{x}} \boldsymbol{C}(\boldsymbol{x})^{T}$. Using the chain rule, the gradient of $f$ with respect to $\boldsymbol{x}$ is given by $\nabla_{\boldsymbol{x}} f(\boldsymbol{C}(\boldsymbol{x}))=\nabla_{\boldsymbol{x}} \boldsymbol{C}(\boldsymbol{x})^{T} \nabla_{\boldsymbol{C}} f(\boldsymbol{C}(\boldsymbol{x}))$. This operator is equivalent to attempting to find the gradient of the weighted sum of $\boldsymbol{C}, \boldsymbol{w}^{T} \boldsymbol{C}$, where $\boldsymbol{w}$ is the constant vector of weights. In the case of a general scalar-valued function $f, \boldsymbol{w}$ would be $\nabla_{\boldsymbol{C}} f(\boldsymbol{C}(\boldsymbol{x}))$ and is treated as a constant. In the case of the variance, $f(\boldsymbol{C})=\sigma_{C}^{2}=\frac{1}{L-1}\left(\boldsymbol{C}-\mu_{C} \mathbf{1}\right)^{T}\left(\boldsymbol{C}-\mu_{C} \mathbf{1}\right)$, therefore $\boldsymbol{w}=\nabla_{\boldsymbol{C}} f(\boldsymbol{C}(\boldsymbol{x}))=\frac{2}{L-1}\left(\boldsymbol{C}-\mu_{C} \mathbf{1}\right)$. And in the case of the standard deviation $\sigma_{C}, \boldsymbol{w}=\frac{1}{(L-1) \sigma_{C}}\left(\boldsymbol{C}-\mu_{C} \mathbf{1}\right)$. This means that computing $\boldsymbol{C}$ is required to form $\boldsymbol{w}$.

By caching $\boldsymbol{u}_{i}=\boldsymbol{K}^{-1} \boldsymbol{f}_{i}$ for all $i$ when computing $\boldsymbol{C}$, one can find the $e^{t h}$ element of $\nabla_{\boldsymbol{x}} \boldsymbol{C}(\boldsymbol{x})^{T} \boldsymbol{w}$ using $\sum_{i=1}^{L}-w_{i} \boldsymbol{u}_{i}^{T} \boldsymbol{K}_{e} \boldsymbol{u}_{i}$, where $w_{i}$ is $i^{t h}$ element of $\boldsymbol{w}$.
Computing $\boldsymbol{u}_{i}^{T} \boldsymbol{K}_{e} \boldsymbol{u}_{i}$ requires constant time complexity, therefore the additional time complexity of computing $\nabla_{\boldsymbol{x}} \boldsymbol{C}(\boldsymbol{x})^{T} \boldsymbol{w}$ after computing $\boldsymbol{C}$ with the direct method is $O\left(L \times n_{E}\right)$. In this case, this is the same complexity as forming the Jacobian first and then multiplying, but in the next algorithms, it will be different.

\subsection{Singular value decomposition}

Much like in the mean compliance calculation, the SVD of $\boldsymbol{F}$ can be computed to find $C_{i}$ for all $i$ more efficiently from $\boldsymbol{K}^{-1} \boldsymbol{U} \boldsymbol{S}$. The number of linear system solves required to compute $\boldsymbol{K}^{-1} \boldsymbol{U} \boldsymbol{S}$ is $n_{s}$, the number of singular values of $\boldsymbol{F}$. The computational cost of computing $C_{i}=\boldsymbol{f}_{i}^{T} \boldsymbol{u}_{i}=\boldsymbol{f}_{i}^{T}\left(\boldsymbol{K}^{-1} \boldsymbol{U} \boldsymbol{S}\right) V^{T}[:, i]$ for all $i$ using $\boldsymbol{K}^{-1} \boldsymbol{U} \boldsymbol{S}$ and $\boldsymbol{V}$ is $O\left(L \times n_{s} \times n_{\text {dofs }}\right)$ when $\boldsymbol{F}$ is dense and $O\left(L \times n_{s} \times n_{\text {loaded }}\right)$ when $\boldsymbol{F}$ is sparse with only $n_{\text {loaded }}$ degrees of freedom loaded. The Jacobian $\nabla_{\rho} \boldsymbol{C}$ can be built by first computing $\boldsymbol{K}^{-1} \boldsymbol{F}$ from the cached $\boldsymbol{K}^{-1} \boldsymbol{U} \boldsymbol{S}$ then using it much like in the exact method without SVD. This has a time complexity of $O\left(\left(n_{s} \times n_{\text {dofs }}+n_{E}\right) \times L\right)$.

However, when interested in $\nabla_{\rho} \boldsymbol{C}^{T} \boldsymbol{w}$ instead, a more efficient approach can be used. Let $\boldsymbol{D}_{\boldsymbol{w}}$ be the diagonal matrix with the vector $\boldsymbol{w}$ on the diagonal.

$$
\begin{aligned}
\nabla_{\boldsymbol{\rho}} \boldsymbol{C}^{T} \boldsymbol{w} & =\nabla_{\boldsymbol{\rho}}\left(\boldsymbol{C}^{T} \boldsymbol{w}\right)=\nabla_{\boldsymbol{\rho}} \operatorname{tr}\left(\boldsymbol{D}_{\boldsymbol{w}} \boldsymbol{F}^{T} \boldsymbol{K}^{-1} \boldsymbol{F}\right) \\
& =\nabla_{\boldsymbol{\rho}} \operatorname{tr}\left(\boldsymbol{V}^{T} \boldsymbol{D}_{\boldsymbol{w}} \boldsymbol{V} \boldsymbol{S} \boldsymbol{U}^{T} \boldsymbol{K}^{-1} \boldsymbol{U} \boldsymbol{S}\right)
\end{aligned}
$$

Let $\boldsymbol{X}=\boldsymbol{V}^{T} \boldsymbol{D}_{\boldsymbol{w}} \boldsymbol{V}$ and $\boldsymbol{Q}=\boldsymbol{K}^{-1} \boldsymbol{U} \boldsymbol{S}$. The partial derivative of the above with respect to $\rho_{e}$ is:

$$
\frac{\partial}{\partial \rho_{e}} \operatorname{tr}\left(\boldsymbol{X} \boldsymbol{Q}^{T} \boldsymbol{S} \boldsymbol{U}^{T} \boldsymbol{K}^{-1} \boldsymbol{U} \boldsymbol{S}\right)=-\operatorname{tr}\left(\boldsymbol{X} \boldsymbol{Q}^{T} \boldsymbol{K}_{e} \boldsymbol{Q}\right)
$$

Note that one can cache $\boldsymbol{Q}=\boldsymbol{K}^{-1} \boldsymbol{U} \boldsymbol{S}$ when finding the function value above to be reused in the sensitivity analysis. Let $\boldsymbol{Y}_{e}=\boldsymbol{Q}^{T} \boldsymbol{K}_{e} \boldsymbol{Q}$. The trace above is therefore $\operatorname{tr}\left(\boldsymbol{X} \boldsymbol{Y}_{e}\right)=\operatorname{tr}\left(\boldsymbol{X}^{T} \boldsymbol{Y}_{e}\right)=\sum_{i=1}^{n_{s}} \sum_{j=1}^{n_{s}} \boldsymbol{X}[i, j] \times$ $\boldsymbol{Y}_{e}[i, j]$. Computing $\boldsymbol{Y}_{e}[i, j]$ from the pre-computed $\boldsymbol{Q}$ requires a constant time complexity for each element $e$, and computing $\boldsymbol{X}$ has a time complexity of $O\left(L \times n_{s}^{2}\right)$. The additional time complexity of computing $\nabla_{\rho} \boldsymbol{C}^{T} \boldsymbol{w}$ using this method is therefore $O\left(\left(n_{E}+L\right) \times n_{s}^{2}\right)$. So if $n_{s} \ll L$, significant computational savings can be made compared to directly computing the Jacobian then doing the matrix-vector multiplication $\nabla_{\rho} \boldsymbol{C}^{T} \boldsymbol{w}$ which has a complexity of $O\left(\left(n_{s} \times n_{\text {dofs }}+n_{E}\right) \times L\right)$.

\section{Maximum compliance constraint}

The maximum compliance constraint can be efficiently handled using the augmented Lagrangian optimization 
Table 5: Summary of the computational cost of the algorithms discussed to calculate the mean compliance and its gradient. \#Lin is the number of linear system solves required.

\begin{tabular}{|l|l|l|l|l|}
\hline \multirow{2}{*}{ Method } & \multirow{2}{*}{ \#Lin } & \multirow{2}{*}{ SVD? } & \multicolumn{2}{|c|}{ Time complexity of additional work } \\
\cline { 4 - 5 } & & & Dense & Sparse \\
\hline \hline Exact-Naive & $L$ & $\boldsymbol{X}$ & $O\left(L \times\left(n_{\text {dofs }}+n_{E}\right)\right)$ & $O\left(L \times\left(n_{\text {loaded }}+n_{E}\right)\right)$ \\
\hline Exact-SVD & $n_{s}$ & $\checkmark$ & $O\left(n_{s} \times\left(n_{\text {dofs }}+n_{E}\right)\right)$ & $O\left(n_{s} \times\left(n_{\text {loaded }}+n_{E}\right)\right)$ \\
\hline
\end{tabular}

Table 6: Summary of the computational cost of the algorithms discussed to calculate the load compliances $\boldsymbol{C}$ as well as $\nabla_{\rho} \boldsymbol{C}^{T} \boldsymbol{w}$ for any vector $\boldsymbol{w}$. \#Lin is the number of linear system solves required. This can be used to compute the variance, standard deviation as well as other scalar-valued functions of $\boldsymbol{C}$. If the full Jacobian is required, the naive method requires the same computational cost as that of computing $\nabla_{\rho} \boldsymbol{C}^{T} \boldsymbol{w}$, and the SVD-based method has a time complexity of $O\left(\left(n_{s} \times n_{\text {dofs }}+n_{E}\right) \times L\right)$ for the additional work other than the linear system solves and SVD.

\begin{tabular}{|l|l|l|l|l|}
\hline \multirow{2}{*}{ Method } & \multirow{2}{*}{ \#Lin } & \multirow{2}{*}{ SVD? } & \multicolumn{2}{|c|}{ Time complexity of additional work } \\
\cline { 4 - 5 } & & & Dense & Sparse \\
\hline \hline Exact-Naive & $L$ & $\boldsymbol{X}$ & $O\left(L \times\left(n_{\text {dofs }}+n_{E}\right)\right)$ & $O\left(L \times\left(n_{\text {loaded }}+n_{E}\right)\right)$ \\
\hline Exact-SVD & $n_{s}$ & $\checkmark$ & $O\left(L \times n_{s} \times n_{\text {dofs }}+\left(n_{E}+L\right) \times n_{s}^{2}\right)$ & $O\left(L \times n_{s} \times n_{\text {loaded }}+\left(n_{E}+L\right) \times n_{s}^{2}\right)$ \\
\hline
\end{tabular}

algorithm (Bertsekas, 1996). Assume the following maximum compliance constrained problem is to be solved for some objective $g(\boldsymbol{x})$ using the augmented Lagrangian algorithm:

$\underset{\boldsymbol{x}}{\operatorname{minimize}} g(\boldsymbol{x})$

subject to

$$
C_{i}=\boldsymbol{f}_{i}^{T} \boldsymbol{K}^{-1} \boldsymbol{f}_{i} \leq C_{t} \quad \forall i \in 1 \ldots L,
$$

$0 \leq x_{e} \leq 1 \quad \forall e \in 1 \ldots n_{E}$

where $C_{t}$ is the maximum compliance allowed. In the augmented Lagrangian algorithm, the problem is transformed as follows:

$\underset{\boldsymbol{x}}{\operatorname{minimize}} L(\boldsymbol{x} ; \boldsymbol{\lambda}, r)$

subject to

$0 \leq x_{e} \leq 1 \quad \forall e \in 1 \ldots n_{E}$

$L(\boldsymbol{x} ; \boldsymbol{\lambda}, r)=g(\boldsymbol{x})+$

$$
\sum_{i=1}^{L}\left(\lambda_{i}\left(C_{i}-C_{t}\right)+r \max \left(C_{i}-C_{t}, 0\right)^{2}\right)
$$

where $\boldsymbol{\lambda}$ is the vector of Lagrangian multipliers $\lambda_{i}$, one for each compliance constraint, and $r$ is the constant coefficient of the quadratic penalty. Solving the above problem using a first-order box constrained algorithm requires the gradient of $L(\boldsymbol{x})$. Writing $L(\boldsymbol{x})$ in vector form:

$L(\boldsymbol{x})=g(\boldsymbol{x})+\boldsymbol{\lambda}^{T}\left(\boldsymbol{C}-C_{t} \mathbf{1}\right)+r \boldsymbol{M}^{\prime} \boldsymbol{M}$ where $M$ is the vector whose $i^{t h}$ element is $\max \left(C_{i}-\right.$ $\left.C_{t}, 0\right)$. The gradient of $L(\boldsymbol{x})$ is given by:

$$
\begin{aligned}
\nabla_{\boldsymbol{x}} L(\boldsymbol{x}) & =\nabla_{\boldsymbol{x}} g+\nabla_{\boldsymbol{x}} \boldsymbol{\rho}^{T}\left(\nabla_{\boldsymbol{\rho}}\left(\boldsymbol{\lambda}^{T}\left(\boldsymbol{C}-C_{t} \mathbf{1}\right)+r \boldsymbol{M}^{\prime} \boldsymbol{M}\right)\right. \\
& =\nabla_{\boldsymbol{x}} g+\nabla_{\boldsymbol{x}} \boldsymbol{\rho}^{T} \nabla_{\boldsymbol{\rho}} \boldsymbol{C}^{T}(\boldsymbol{\lambda}+2 \boldsymbol{M})
\end{aligned}
$$

As shown in the previous sections, calculating the product $\nabla_{\rho} \boldsymbol{C}^{T}(\boldsymbol{\lambda}+2 \boldsymbol{M})$ can be done efficiently by finding the gradient $\nabla_{\boldsymbol{\rho}}\left(\boldsymbol{C}^{T} \boldsymbol{w}\right)$ using $\boldsymbol{w}=(\boldsymbol{\lambda}+2 \boldsymbol{M})$. Therefore, the results from Table 6 apply.

\section{Setup and Implementation}

In this section, the most important implementation details and algorithm settings used in the experiments are presented.

\subsection{Test problems}

\subsubsection{D cantilever beam}

The 2D cantilever beam problem shown in Figure 1 was used to run the experiments. A ground mesh of plane stress quadrilateral elements was used, where each element is a square of side length $1 \mathrm{~mm}$, and a sheet thickness of $1 \mathrm{~mm}$. Linear iso-parametric interpolation functions were used for the field and geometric basis functions. A Young's modulus of $1 \mathrm{MPa}$ and Poisson's ratio of 0.3 were used. Finally, a chequerboard density filter for unstructured meshes was used with a radius of $2 \mathrm{~mm}$ Huang and Xie (2010). A 3D version of the problem above was also solved. Details of the $3 \mathrm{D}$ problem and the results are shown in the appendix. 


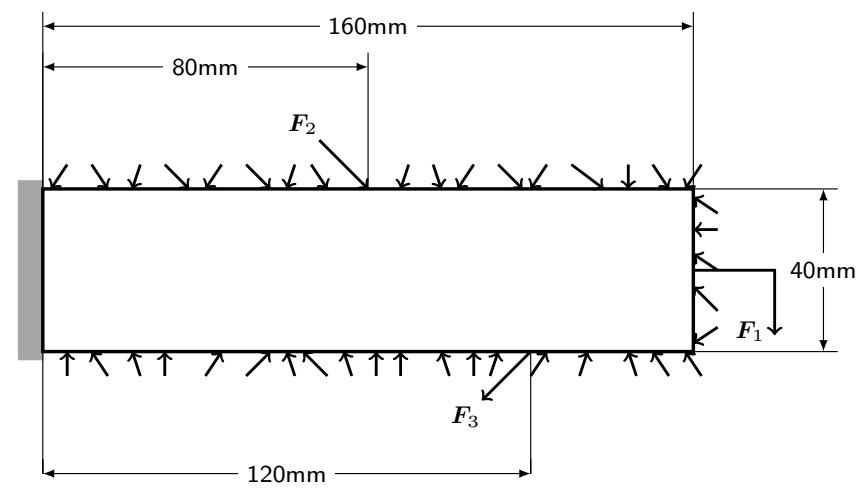

Fig. 1: Cantilever beam problem. $\boldsymbol{F}_{2}$ and $\boldsymbol{F}_{3}$ are at 45 degree angles.

Three variants of the cantilever beam problem were solved:

1. Minimization of the mean compliance $\mu_{C}$ subject to a volume constraint with a volume fraction of 0.4 ,

2. Minimization of a weighted sum of the mean and standard deviation (mean-std) of the compliance $\mu_{C}+2.0 \sigma_{C}$ subject to a volume constraint with a volume fraction of 0.4 , and

3. Volume minimization subject to a maximum compliance constraint with a compliance threshold of $70000 \mathrm{Nmm}$.

A total of 1000 load scenarios were sampled from:

$\boldsymbol{f}_{i}=s_{1} \boldsymbol{F}_{1}+s_{2} \boldsymbol{F}_{2}+s_{3} \boldsymbol{F}_{3}+\frac{1}{7} \sum_{j=4}^{10} s_{j} \boldsymbol{F}_{j}$

where $\boldsymbol{F}_{1}, \boldsymbol{F}_{2}$ and $\boldsymbol{F}_{3}$ are unit vectors with directions as shown in Figure 1, $\boldsymbol{F}_{2}$ and $\boldsymbol{F}_{3}$ are at 45 degrees. $s_{1}$, $s_{2}$ and $s_{3}$ are identically and independently uniformly distributed random variables between -2 and 2. $\boldsymbol{F}_{j}$ for $j$ in $4 \ldots 10$ are vectors with non-zeros at all the surface degrees of freedom without a Dirichlet boundary condition. The non-zero values are identically and independently normally distributed random variables with mean 0 and standard deviation $1 . s_{j}$ for $j$ in $4 \ldots 10$ are also identically and independently normally distributed random variables with mean 0 and standard deviation 1. The same loading scenarios were used for the 3 test problems. Let $\boldsymbol{F}$ be the matrix whose columns are the sampled $\boldsymbol{f}_{i}$ vectors. The way the loading scenarios are defined, the rank of $\boldsymbol{F}$ can be at most 10 and was actually exactly 10 in our experiments. Given the low rank structure of $\boldsymbol{F}$, the SVD approaches should be expected to be significantly more efficient than their naive counterparts.

\subsubsection{D cantilever beam}

A $3 \mathrm{D}$ version of the $2 \mathrm{D}$ cantilever beam test problem above was also solved using the methods proposed. A $60 \mathrm{~mm}$ × $20 \mathrm{~mm}$ x $20 \mathrm{~mm}$ 3D cantilever beam was used with hexahedral elements of cubic shape and side length of $1 \mathrm{~mm}$. The loads $\boldsymbol{F}_{1}, \boldsymbol{F}_{2}$ and $\boldsymbol{F}_{3}$ were positioned at $(60,10,10),(30,20,10)$ and $(40,0,10)$ where the coordinates represent the length, height and depth respectively. The remaining loads and multipliers were sampled from the same distributions as the $2 \mathrm{D}$ problem. A density filter radius of $3 \mathrm{~mm}$ was also used for the $3 \mathrm{D}$ problem.

\subsection{Software}

All the topology optimization algorithms described in this paper were implemented in TopOpt.jl 11 using the Julia programming language (Bezanson et al. 2014) version 1.3 for handling generic unstructured, iso-parametric meshes.

\subsection{Settings}

The value of $x_{\min }$ used was 0.001 for all problems and algorithms. Penalization was done prior to interpolation to calculate $\boldsymbol{\rho}$ from $\boldsymbol{x}$. A power penalty function and a regularized Heaviside projection were used. All of the problems were solved using 2 continuation SIMP routines. The first incremented the penalty value from $p=1$ to $p=6$ in increments of 0.5 . Then the Heaviside projection parameter $\beta$ was incremented from $\beta=0$ to $\beta=20$ in increments of 4 keeping the penalty value fixed at 6 . An exponentially decreasing tolerance from $1 e-3$ to $1 e-4$ was used for both continuations.

The mean and mean-std compliance minimization SIMP subproblems problems were solved using the method of moving asymptotes (MMA) algorithm Svanberg (1987). MMA parameters of $s_{\text {init }}=0.5, s_{\text {incr }}=1.1$ and $s_{\text {decr }}=$ 0.7 were used as defined in the MMA paper with a maximum of 1000 iterations for each subproblem. The dual problem of the convex approximation was solved using a log-barrier box-constrained nonlinear optimization solver, where the barrier problem was solved using the nonlinear CG algorithm for unconstrained nonlinear optimization (Nocedal and Wright, 2006) as implemented in Optim.jl $\left.\right|^{2}$ (K Mogensen and N Riseth. 2018). The nonlinear CG itself used the line search algorithm from Hager and Zhang (2006) as implemented

\footnotetext{
1 https://github.com/mohamed82008/TopOpt.jl

2 https://github.com/JuliaNLSolvers/Optim.jl
} 
in LineSearches.jl ${ }^{3}$. The stopping criteria used was the one adopted by the KKT solver, IPOPT (Wächter and Biegler, 2006). This stopping criteria is less scale sensitive than the KKT residual as it scales down the residual by a value proportional to the mean absolute value of the Lagrangian multipliers.

The maximum compliance constrained SIMP subproblems were solved using a primal-dual augmented Lagrangian method (Bertsekas, 1996). The inequality constraints were relaxed resulting in a box constrained max-min primal-dual problem. A projected gradient descent algorithm was used for the primal and dual problems with a backtracking line search. The maximum step size of the line search was initialized to 1 and adapted to be 1.5 the step size of the previous line search for both the primal and dual problems. A total of 10 dual iterations were used with a maximum of 50 primal iterations per dual iteration. The IPOPT termination criteria above was also used here. To regularize the scale of the problem, the compliance constraints were divided by the maximum compliance at the full ground mesh design. A trust region of 0.1 was used. An initial quadratic penalty coefficient of 0.1 was used with a growth factor of 3 in every dual iteration. Finally, an initial solution of 1.0 for all the primal variables and 1 for all the Lagrangian multipliers was used.

\subsection{Replication of Results}

The instructions and codes needed to replicate the results in this paper are given in https://github.com/ mohamed82008/RobustComplianceCode

\section{Results and Discussion}

\subsection{Speed comparison}

Tables 7 and 8 show the values computed for the mean compliance $\mu_{C}$ and its standard deviation $\sigma_{C}$ respectively together with the time required to compute their values and gradients using: the exact naive approach (Exact-Naive) and the exact method with SVD (ExactSVD). As expected, the proposed exact SVD approach computes the exact mean compliance or its standard deviation and their gradient in a small fraction of the time it takes to compute them using the naive approaches.

3 https://github.com/JuliaNLSolvers/LineSearches.jl
Table 7: The table shows the function values of $\mu_{C}$ computed using the naive exact method (Exact-Naive) and the exact method with SVD (Exact-SVD). The table also shows the time required to compute $\mu_{C}$ and its gradient in each case.

\begin{tabular}{|c|c|c|}
\hline Method & $\mu_{C}(\mathrm{Nmm})$ & Time $(\mathrm{s})$ \\
\hline \hline Exact-Naive & 3328.7 & 24.2 \\
\hline Exact-SVD & 3328.7 & 0.4 \\
\hline
\end{tabular}

Table 8: The table shows the function values of $\sigma_{C}$ and its gradients for a full ground mesh computed using the naive exact method (Exact-Naive) and the exact method with SVD (Exact-SVD). The table also shows the time required to compute $\sigma_{C}$ and its gradient in each case.

\begin{tabular}{|c|c|c|}
\hline Method & $\sigma_{C}(\mathrm{Nmm})$ & Time $(\mathrm{s})$ \\
\hline \hline Exact-Naive & 4172.8 & 28.0 \\
\hline Exact-SVD & 4172.8 & 1.5 \\
\hline
\end{tabular}

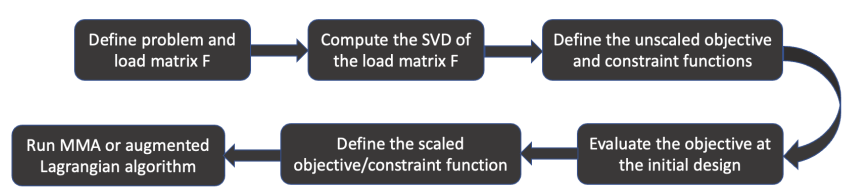

Fig. 2: Flowchart of the experiments' workflow. Only the mean compliance objective, mean-std compliance objective or maximum compliance constraint are scaled by the inverse of their initial value. The volume function is not scaled.

\subsection{Optimization}

In this section, a number of stochastic, risk-averse and robust compliance-based optimization problems are solved using the proposed methods. Figure 2 shows the experiments' workflow.

\subsubsection{Mean compliance minimization}

To demonstrate the effectiveness of the proposed approaches, the $2 \mathrm{D}$ and $3 \mathrm{D}$ cantilever beam problems described in section 5 were solved using the proposed SVD-based methods. Table 9 shows the statistics of the final optimal solutions obtained by minimizing the mean compliance subject to the volume fraction constraint using the SVD-based method to evaluate the mean compliance. The optimal topologies are shown in Figures 3 and 4 . 


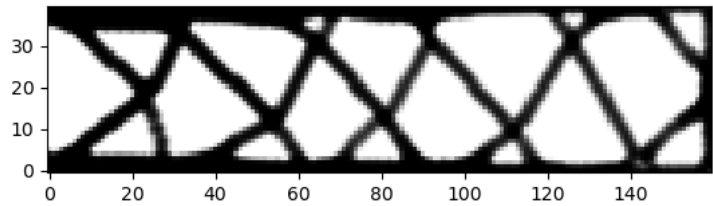

Fig. 3: Optimal topology of the mean compliance minimization problem using continuation SIMP and the SVD-based method for evaluating the mean compliance.

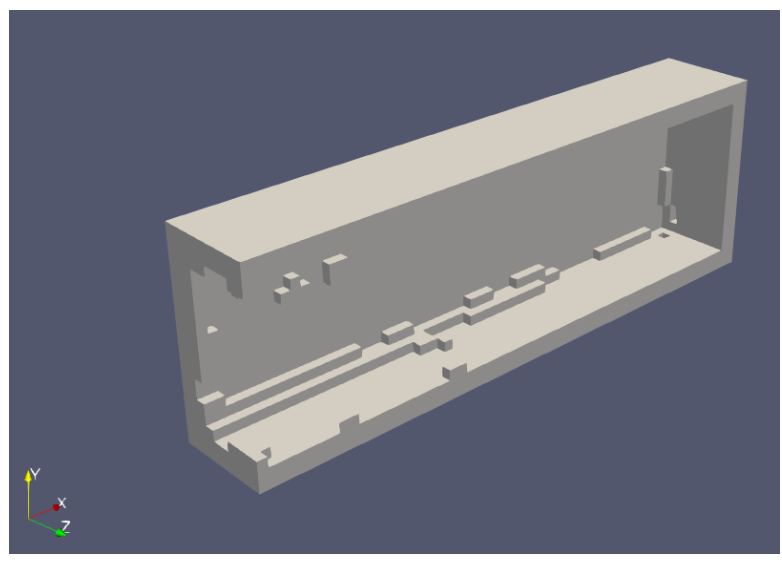

(a) Left half

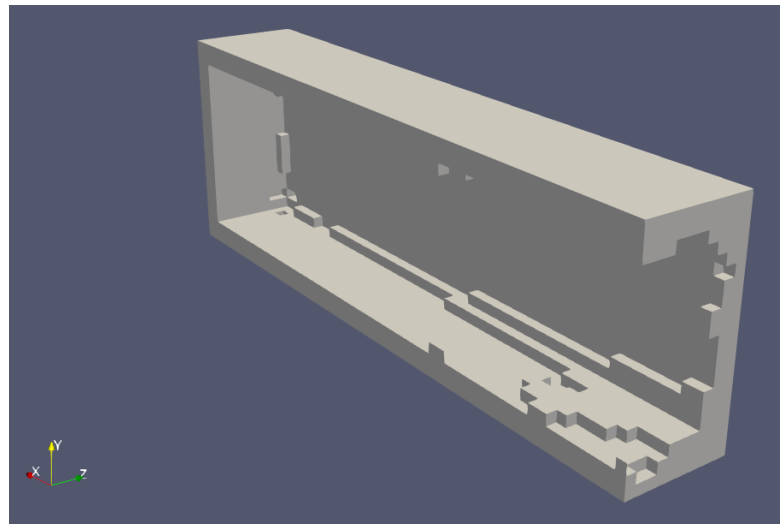

(b) Right half

Fig. 4: Cut views of the optimal topologies of the 3D mean compliance minimization problem using exact method with SVD.
Table 9: Summary statistics of the load compliances of the optimal solutions of the 2D and 3D mean compliance minimization problems using the SVD-based method to evaluate the mean compliance.

\begin{tabular}{|c|c|c|}
\hline Compliance Stat & $2 \mathrm{D}$ & $3 \mathrm{D}$ \\
\hline \hline$\mu_{C}(\mathrm{Nmm})$ & 9392.8 & 22072.1 \\
\hline$\sigma_{C}(\mathrm{Nmm})$ & 9688.8 & 16628.7 \\
\hline$C_{\max }(\mathrm{Nmm})$ & 125455.0 & 184055.0 \\
\hline$C_{\min }(\mathrm{Nmm})$ & 467.9 & 1785.8 \\
\hline$V$ & 0.400 & 0.400 \\
\hline Time $(\mathrm{s})$ & 491.5 & 3849.6 \\
\hline
\end{tabular}

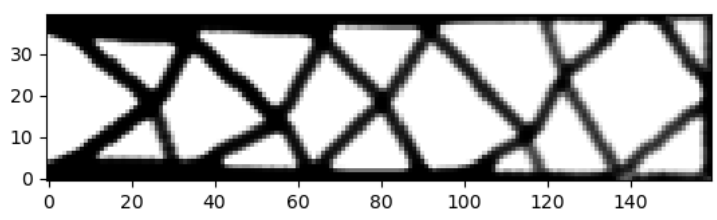

Fig. 5: Optimal topology of the mean-std compliance minimization problem using continuation SIMP and the SVD-based method to compute the mean-std.

\subsubsection{Mean-std compliance minimization}

Similarly, Table 10 shows the statistics of the final solutions of the 2D and 3D mean-std minimization problems solved using the SVD-based method. The optimal topologies are shown in Figures 5 and 6. The algorithm converged to reasonable, feasible designs. Additionally, as expected the mean-std minimization algorithm converged to solutions with lower compliance standard deviations but higher mean compliances compared to the mean minimization algorithm.

To highlight the effect of the multiple $m$ of the standard deviation in the objective $\mu_{C}+m \times \sigma_{C}$, the same problem was solved for different values of $m$. Figure 7 shows the profile of the mean and standard deviation of the compliance. Interestingly due to the non-convexity of the problem, increasing the standard deviation's multiple can sometimes lead to a simultaneous increase or reduction in the mean and standard deviation of the compliance. The different optimal topologies are shown in Figure 8 , 


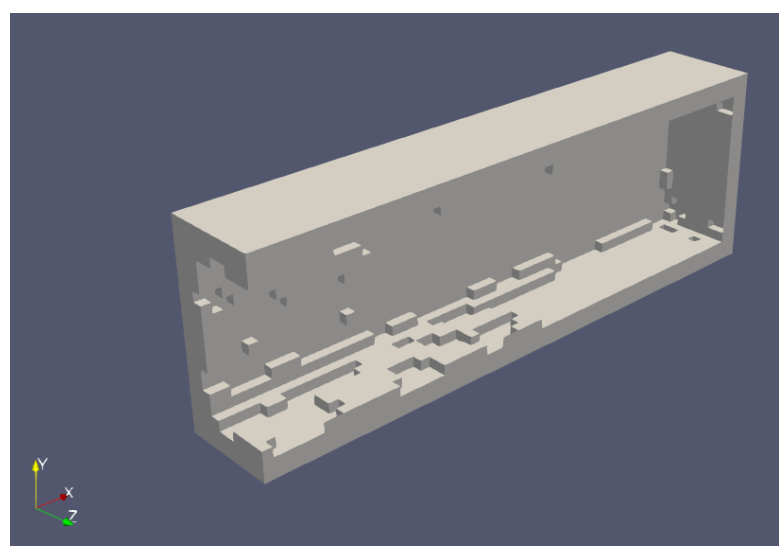

(a) Left half

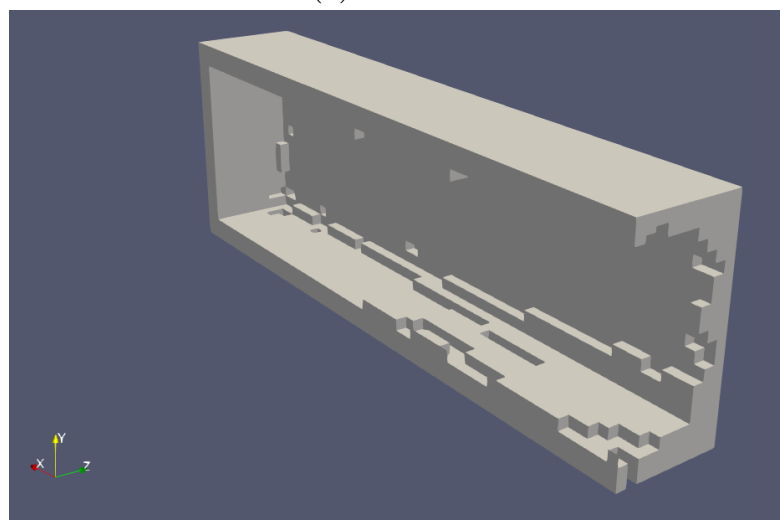

(b) Right half

Fig. 6: Cut views of the optimal topologies of the 3D mean-std compliance minimization problem using the exact method with SVD.

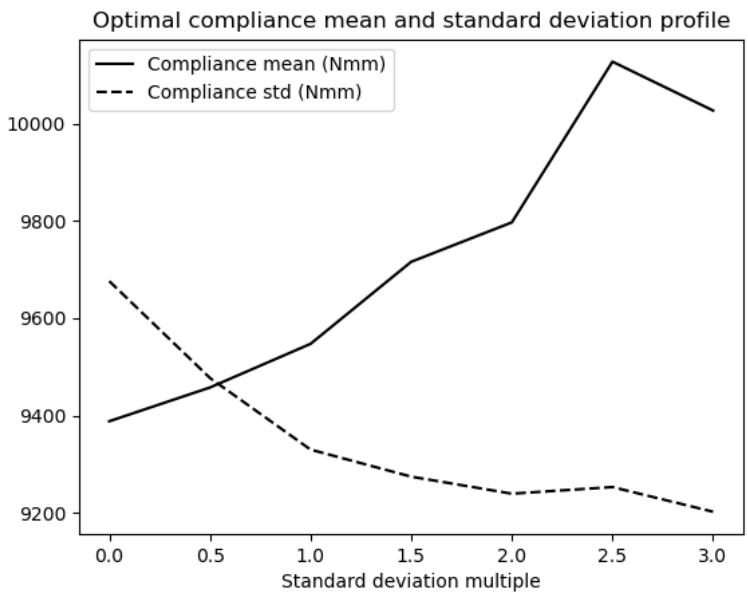

Fig. 7: Profile of the optimal mean and standard deviation of the compliance for different standard deviation multiples in the objective.
Table 10: Summary statistics of the load compliances of the optimal solutions of the $2 \mathrm{D}$ and $3 \mathrm{D}$ mean-std compliance minimization problems using the SVD-based method to evaluate the mean-std compliance.

\begin{tabular}{|c|c|c|}
\hline Compliance Stat & $2 \mathrm{D}$ & $3 \mathrm{D}$ \\
\hline \hline$\mu_{C}(\mathrm{Nmm})$ & 9796.9 & 22216.7 \\
\hline$\sigma_{C}(N \mathrm{~mm})$ & 9240.0 & 16220.2 \\
\hline$\mu_{C}+2.0 \sigma_{C}(N m m)$ & 28283.7 & 54848.8 \\
\hline$C_{\max }(\mathrm{Nmm})$ & 117883.1 & 176153.2 \\
\hline$C_{\min }(\mathrm{Nmm})$ & 527.7 & 1872.0 \\
\hline$V$ & 0.400 & 0.400 \\
\hline Time $(\mathrm{s})$ & 229.8 & 3528.2 \\
\hline
\end{tabular}

\subsubsection{Maximum compliance constrained optimization}

The 2D and 3D maximum compliance constrained volume minimization problems were solved using the SVDbased approach. The 2D optimal topology, shown in Figure 9, had a volume fraction of 0.584 and a maximum compliance of $69847.0 \mathrm{Nmm}$ and was reached in 662.7 s. The 3D optimal topology, shown in Figure 10. had a volume fraction of 0.791 and a maximum compliance of $68992.4 \mathrm{Nmm}$ and was reached in 43740.6 s.

\section{Conclusion}

In this paper, a number of exact methods were proposed to handle load uncertainty in compliance topology optimization problems where the uncertainty is described in the form of a set of finitely many loading scenarios. By exploiting low rank structures in loading scenarios, significant performance improvements were achieved using novel SVD-based methods. Such improvement was demonstrated via complexity analysis and computational experiments. The methods presented here are fundamentally data-driven in the sense that no probability distributions or domains are assumed for the loading scenarios. This sets this work apart from most of the literature in the domain of stochastic and robust topology optimization where a distribution or domain is assumed. Additionally, the methods proposed here were shown to be particularly suitable with the augmented Lagrangian algorithm when dealing with maximum compliance constraints.

\section{Acknowledgments}

This research did not receive any specific grant from funding agencies in the public, commercial, or not-forprofit sectors. 


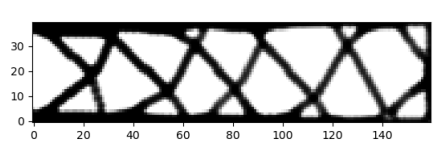

(a) $m=0$

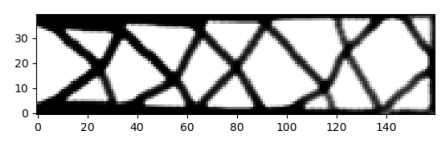

(d) $m=1.5$

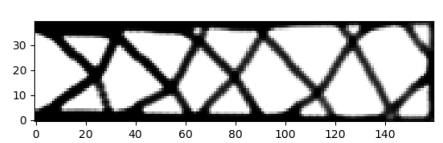

(b) $m=0.5$

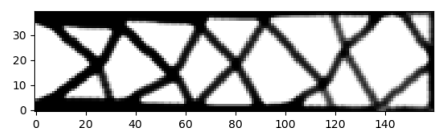

(e) $m=2.0$

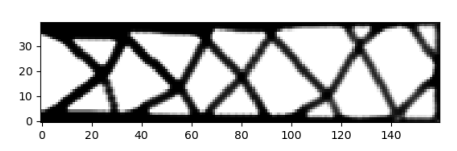

(c) $m=1.0$

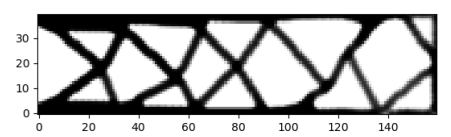

(f) $m=2.5$

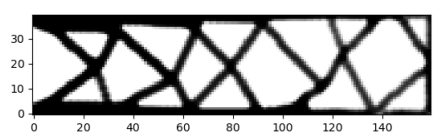

(g) $m=3.0$

Fig. 8: Optimal topologies of the 2D mean-std compliance minimization problem using different standard deviation multiples $m$ in the objective $\mu_{C}+m \sigma_{C}$.

\section{Conflict of Interest}

The authors have no conflict of interest to declare.

\section{A Partial derivative of the inverse quadratic form}

In this section, it will be shown that the $i^{\text {th }}$ partial derivative of:

$$
f(\boldsymbol{x})=\boldsymbol{v}^{T}(\boldsymbol{A}(\boldsymbol{x}))^{-1} \boldsymbol{v}
$$

is

$$
\frac{\partial f}{\partial x_{i}}=-\boldsymbol{y}^{T} \frac{\partial \boldsymbol{A}}{\partial x_{i}} \boldsymbol{y}^{T}
$$

where $\boldsymbol{A}$ is a matrix-valued function of $\boldsymbol{x}, \boldsymbol{v}$ is a constant vector and $\boldsymbol{y}=\boldsymbol{A}^{-1} \boldsymbol{v}$ is a an implicit function of $\boldsymbol{x}$ because $\boldsymbol{A}$ is a function of $\boldsymbol{x}$. 


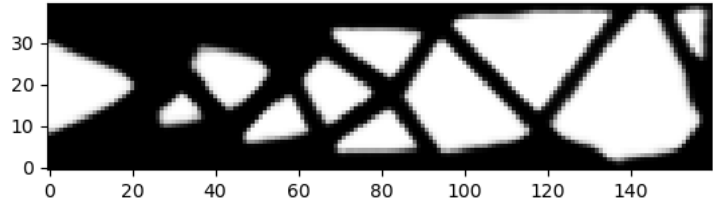

Fig. 9: Optimal topology of the volume minimization problem subject to a maximum compliance constraint using continuation SIMP and the augmented Lagrangian method with the exact SVD approach. The maximum compliance of the design above is 69847.0 $\mathrm{Nmm}$ and the volume fraction is 0.584 .

$$
\begin{aligned}
\boldsymbol{v} & =\boldsymbol{A} \boldsymbol{y} \\
\mathbf{0} & =\boldsymbol{A} \frac{\partial \boldsymbol{y}}{\partial x_{i}}+\frac{\partial \boldsymbol{A}}{\partial x_{i}} \boldsymbol{y} \\
\frac{\partial y}{\partial x_{i}} & =-\boldsymbol{A}^{-1} \frac{\partial \boldsymbol{A}}{\partial x_{i}} \boldsymbol{y} \\
f(\boldsymbol{x}) & =\boldsymbol{v}^{T} \boldsymbol{A}^{-1} \boldsymbol{v} \\
& =\boldsymbol{y}^{T} \boldsymbol{A} \boldsymbol{y} \\
\frac{\partial f}{\partial x_{i}} & =2 \boldsymbol{y}^{T} \boldsymbol{A} \frac{\partial \boldsymbol{y}}{\partial x_{i}}+\boldsymbol{y}^{T} \frac{\partial \boldsymbol{A}}{\partial x_{i}} \boldsymbol{y} \\
& =-2 \boldsymbol{y}^{T} \boldsymbol{A} \boldsymbol{A}^{-1} \frac{\partial \boldsymbol{A}}{\partial x_{i}} \boldsymbol{y}+\boldsymbol{y}^{T} \frac{\partial \boldsymbol{A}}{\partial x_{i}} \boldsymbol{y} \\
& =-2 \boldsymbol{y}^{T} \frac{\partial \boldsymbol{A}}{\partial x_{i}} \boldsymbol{y}+\boldsymbol{y}^{T} \frac{\partial \boldsymbol{A}}{\partial x_{i}} \boldsymbol{y} \\
& =-\boldsymbol{y}^{T} \frac{\partial \boldsymbol{A}}{\partial x_{i}} \boldsymbol{y}
\end{aligned}
$$

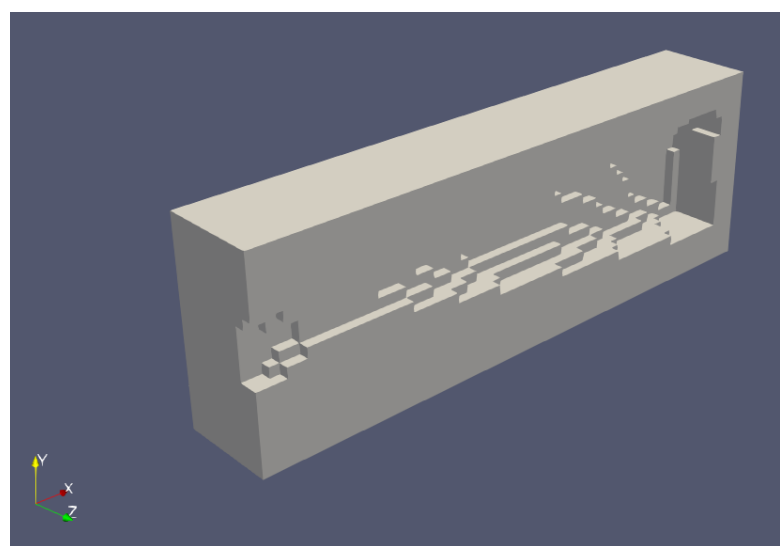

(a) Left half

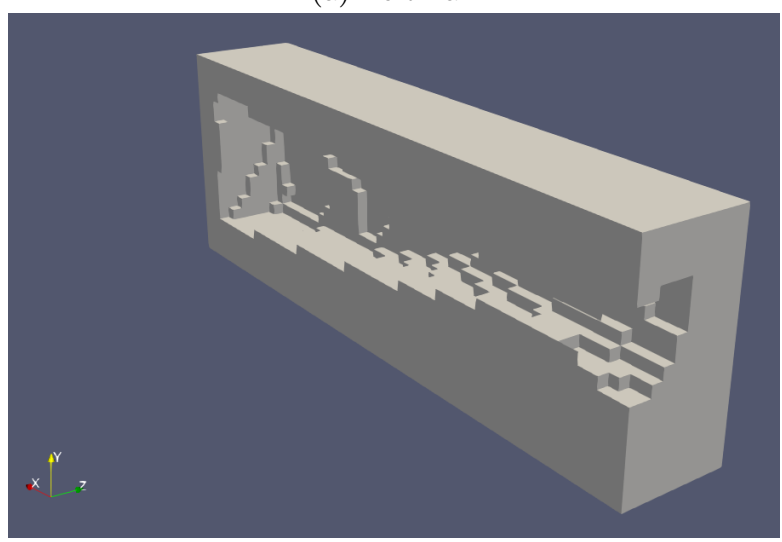

(b) Right half

Fig. 10: Cut views of the 3D optimal topology of the volume minimization problem subject to a maximum compliance constraint using continuation SIMP and the augmented Lagrangian method with the exact SVD approach. The maximum compliance of the design above is $68992.4 \mathrm{Nmm}$ and the volume fraction is 0.791 .

\section{References}

Aharon Ben-Tal, Laurent El Ghaoui, Nemirovski A (2009) Robust Optimization. Princeton University Press

Bendsoe MP (1989) Optimal shape design as a material distribution problem. Structural Optimization 1(4):193-202

Bendsoe MP, Sigmund O (2004) Topology Optimization: Theory, Methods and Applications., 2nd edn. SpringerVerlag Berlin Heidelberg, DOI 10.1007/978-3-662-05086-6

Bertsekas DP (1996) Constrained Optimization and Lagrange Multiplier Methods. Athena Scientific

Bertsimas D, Brown DB, Caramanis C (2011) Theory and applications of robust optimization. SIAM Review 53(3):464501, DOI 10.1137/080734510

Bezanson J, Edelman A, Karpinski S, Shah VB (2014) Julia: A Fresh Approach to Numerical Computing

Brittain K, Silva M, Tortorelli DA (2012) Minmax topology optimization. Structural and Multidisciplinary Optimization 45(5):657-668, DOI 10.1007/s00158-011-0715-y

Chen S, Lee S, Chen W (2010) Level set based robust shape and topology optimization under random field uncertainties. Proceedings of the ASME International Design En- 
gineering Technical Conferences and Computers and Information in Engineering Conference 2009, DETC2009 5(PART B):1295-1305, DOI 10.1115/DETC2009-87083

Choi SK, Grandhi RV, Canfield RA (2007) Reliabilitybased Structural Design. Springer-Verlag, DOI 10.1017/ CBO9781107415324.004, arXiv: 1011.1669v3

Cuellar N, Pereira A, Menezes IF, Cunha A (2018) Nonintrusive polynomial chaos expansion for topology optimization using polygonal meshes. Journal of the Brazilian Society of Mechanical Sciences and Engineering 40(12):118, DOI 10.1007/s40430-018-1464-2, URL https://doi. org/10.1007/s40430-018-1464-2

Dunning PD, Kim HA (2013) Robust topology optimization: Minimization of expected and variance of compliance. AIAA Journal 51(11):2656-2664, DOI 10.2514/1.J052183

Dunning PD, Kim HA, Mullineux G (2011) Introducing loading uncertainty in topology optimization. AIAA Journal 49(4):760-768, DOI 10.2514/1.J050670

Garcia-Lopez NP, Sanchez-Silva M, Medaglia AL, Chateauneuf A (2013) An improved robust topology optimization approach using multiobjective evolutionary algorithms. Computers and Structures 125:1-10, DOI 10.1016/j.compstruc.2013.04.025, URL http://dx.doi.org/10.1016/j.compstruc.2013.04.025

Guest JK, Igusa T (2008) Structural optimization under uncertain loads and nodal locations. Computer Methods in Applied Mechanics and Engineering 198(1):116-124, DOI 10.1016/j.cma.2008.04.009, URL http://dx.doi.org/10. $1016 / \mathrm{j} . \mathrm{cma} .2008 .04 .009$

Guest JK, Prévost JH, Belytschko T (2004) Achieving minimum length scale in topology optimization using nodal design variables and projection functions. International Journal for Numerical Methods in Engineering 61(2):238-254, DOI $10.1002 /$ nme.1064

Guo SX, Lu ZZ (2015) A non-probabilistic robust reliability method for analysis and design optimization of structures with uncertain-but-bounded parameters. Applied Mathematical Modelling 39(7):1985-2002, DOI 10.1016/ j.apm.2014.10.026, URL http://dx.doi.org/10.1016/j. apm.2014.10.026

Hager WW, Zhang H (2006) Algorithm 851: CG_DESCENT, a conjugate gradient method with guaranteed descent. ACM Transactions on Mathematical Software (TOMS) 32(1):113-137, DOI 10.1145/1132973.1132979, URL http: //portal.acm.org/citation.cfm?id=1132979

Holmberg E, Thore CJ, Klarbring A (2015) Worst-case topology optimization of self-weight loaded structures using semi-definite programming. Structural and Multidisciplinary Optimization 52(5):915-928, DOI 10.1007/ s00158-015-1285- 1

Huang X, Xie YM (2010) A further review of ESO type methods for topology optimization. Structural and Multidisciplinary Optimization 41(5):671-683

Hutchinson MF (1990) A stochastic estimator of the trace of the influence matrix for laplacian smoothing splines. Communications in Statistics - Simulation and Computation 19(2):433-450, DOI 10.1080/03610919008812866

Jalalpour M, Tootkaboni M (2016) An efficient approach to reliability-based topology optimization for continua under material uncertainty. Structural and Multidisciplinary Optimization 53(4):759-772, DOI 10.1007/s00158-015-1360-7

Jung HS, Cho S (2004) Reliability-based topology optimization of geometrically nonlinear structures with loading and material uncertainties. Finite Elements in Analysis and Design 41(3):311-331, DOI 10.1016/j.finel.2004.06.002
K Mogensen P, N Riseth A (2018) Optim: A mathematical optimization package for Julia. Journal of Open Source Software 3(24):615, DOI 10.21105/joss.00615, URL http: //joss.theoj.org/papers/10.21105/joss.00615, arXiv: 1710.07708

Kang Z, Luo Y (2009) Non-probabilistic reliability-based topology optimization of geometrically nonlinear structures using convex models. Computer Methods in Applied Mechanics and Engineering 198(41-44):3228-3238, DOI 10.1016/j.cma.2009.06.001, URL http://dx.doi.org/10. $1016 / \mathrm{j}$.cma.2009.06.001

Keshavarzzadeh V, Fernandez F, Tortorelli DA (2017) Topology optimization under uncertainty via non-intrusive polynomial chaos expansion. Computer Methods in Applied Mechanics and Engineering 318:120-147, DOI 10.1016/ j.cma.2017.01.019, URL http://dx.doi.org/10.1016/j. cma.2017.01.019

Kharmanda G, Olhoff N (2002) Reliability-Based Topology Optimization as a New Strategy to Generate Different Structural Topologies. 15th Nordic Seminar on Computational Mechanics (January)

Kharmanda G, Olhoff N, Mohamed A, Lemaire M (2004) Reliability-based topology optimization. Structural and Multidisciplinary Optimization 26(5):295-307, DOI 10. 1007/s00158-003-0322-7

Kim C, Wang S, Bae KR, Moon H, Choi KK (2006) Reliability-based topology optimization with uncertainties. Journal of Mechanical Science and Technology 20(4):494504, DOI 10.1007/BF02916480

Kim SR, Park JY, Lee WG, Yu JS, Han SY (2007) ReliabilityBased Topology Optimization Based on Evolutionary Structural Optimization. International Journal of Mechanical Systems Science and Engineering 1(3):168-172

Kim SR, Lee WG, Park JY, Yu JS, Han SY (2008) Reliabilitybased topology optimization using reliability index approach. ICEM 2008: International Conference on Experimental Mechanics 2008 7375(August 2009):73752W, DOI $10.1117 / 12.839233$

Kriegesmann B, Lüdeker JK (2019) Robust compliance topology optimization using the first-order second-moment method. Structural and Multidisciplinary Optimization 60(1):269-286, DOI 10.1007/s00158-019-02216-8

Liu J, Wen G (2018) Continuum topology optimization considering uncertainties in load locations based on the cloud model. Engineering Optimization 50(6):1041-1060, DOI 10.1080/0305215X.2017.1361417, URL https://doi.org/ 10.1080/0305215X.2017.1361417

Liu JT, Gea HC (2018) Robust topology optimization under multiple independent unknown-but-bounded loads. Computer Methods in Applied Mechanics and Engineering 329:464-479, DOI 10.1016/j.cma.2017.09.033, URL https: //doi.org/10.1016/j.cma.2017.09.033

Luo Y, Kang Z, Luo Z, Li A (2009) Continuum topology optimization with non-probabilistic reliability constraints based on multi-ellipsoid convex model. Structural and Multidisciplinary Optimization 39(3):297-310, DOI 10.1007/s00158-008-0329-1

Martínez-Frutos J, Herrero-Pérez D (2016) Large-scale robust topology optimization using multi-GPU systems. Computer Methods in Applied Mechanics and Engineering 311:393-414, DOI 10.1016/j.cma.2016.08.016

Martínez-Frutos J, Herrero-Pérez D, Kessler M, Periago F (2018) Risk-averse structural topology optimization under random fields using stochastic expansion methods. Computer Methods in Applied Mechanics and Engineering 330:180-206, DOI 10.1016/j.cma.2017.10.026, URL https : 
//doi.org/10.1016/j.cma.2017.10.026

Nguyen TH, Song J, Paulino GH (2011) Single-loop system reliability-based topology optimization considering statistical dependence between limit-states. Structural and Multidisciplinary Optimization 44(5):593-611, DOI 10.1007/ s00158-011-0669-0

Nocedal J, Wright SJ (2006) Numerical Optimization. Springer Sc, DOI 10.1002/lsm.21040, NIHMS150003

Ouyang G, Zhang X, Kuang Y (2008) Reliability-based topology optimization of continuous structures. Proceedings of the World Congress on Intelligent Control and Automation (WCICA) (50375051):7021-7025, DOI 10.1109/WCICA. 2008.4594005

Rojas-Labanda S, Stolpe M (2015) Automatic penalty continuation in structural topology optimization. Structural and Multidisciplinary Optimization 52(6):1205-1221

Shapiro A, Dentcheva D, Ruszczyński A (2009) Lectures on Stochastic Programming. DOI 10.1137/1.9780898718751

Sigmund O (2001) A 99 line topology optimization code written in matlab. Structural and Multidisciplinary Optimization 21(2):120-127

Silva M, Tortorelli DA, Norato JA, Ha C, Bae HR (2010) Component and system reliability-based topology optimization using a single-loop method. Structural and Multidisciplinary Optimization 41(1):87-106, DOI 10.1007/ s00158-009-0401-5

Svanberg K (1987) The method of moving asymptotes - a new method for structural optimization. International Journal for Numerical Methods in Engineering 24(2):359-373

Thore CJ, Holmberg E, Klarbring A (2017) A general framework for robust topology optimization under loaduncertainty including stress constraints. Computer Methods in Applied Mechanics and Engineering 319:1-18, DOI 10.1016/j.cma.2017.02.015, URL http://dx.doi.org/10. 1016/j.cma.2017.02.015

Tu J, Choi KK, Park YH (1999) A new study on reliabilitybased design optimization. Journal of Mechanical Design, Transactions of the ASME 121(4):557-564, DOI 10.1115/ 1.2829499

Wächter A, Biegler LT (2006) On the Implementation of a Primal-Dual Interior Point Filter Line Search Algorithm for Large-Scale Nonlinear Programming, vol 106

Wang L, Liu D, Yang Y, Wang X, Qiu Z (2017) A novel method of non-probabilistic reliability-based topology optimization corresponding to continuum structures with unknown but bounded uncertainties. Computer Methods in Applied Mechanics and Engineering 326:573-595, DOI 10.1016/j.cma.2017.08.023, URL http://dx.doi.org/10. $1016 / \mathrm{j} . \mathrm{cma} .2017 .08 .023$

Wang L, Liang J, Wu D (2018) A non-probabilistic reliabilitybased topology optimization (NRBTO) method of continuum structures with convex uncertainties. Structural and Multidisciplinary Optimization 58(6):2601-2620, DOI 10.1007/s00158-018-2040-1

Wang L, Liu D, Yang Y, Hu J (2019a) Novel methodology of Non-probabilistic Reliability-based Topology Optimization (NRBTO) for multi-material layout design via interval and convex mixed uncertainties. Computer Methods in Applied Mechanics and Engineering 346:550-573, DOI 10.1016/j. cma.2018.11.035, URL https://doi.org/10.1016/j.cma. 2018.11.035

Wang L, Xia H, Zhang X, Lv Z (2019b) Non-probabilistic reliability-based topology optimization of continuum structures considering local stiffness and strength failure. Computer Methods in Applied Mechanics and Engineering 346:788-809, DOI 10.1016/j.cma.2018.09.021, URL https : //doi.org/10.1016/j.cma.2018.09.021

Xie Y, Steven G (1992) Shape and layout optimization via an evolutionary procedure. In: Proceedings of the International Conference Comput. Eng. (Hong Kong), Hong Kong University, p 421

Yang X, Xie Y, Steven G, Querin O (1998) Bi-directional evolutionary structural optimization. In: Proceedings of the 7th AIAA/USAF/NASA/ISSMO Symposium Multidisc Anal. Optim (St. Louis), pp 1449-1457

Yin H, Yu D, Xia B (2018) Reliability-based topology optimization for structures using fuzzy set model. Computer Methods in Applied Mechanics and Engineering 333:197217, DOI 10.1016/j.cma.2018.01.019, URL https://doi. org $/ 10.1016 / \mathrm{j} . \mathrm{cma} .2018 .01 .019$

Youn BD, Choi KK (2004) Selecting probabilistic approaches for realiability-based design optimization. AIAA Journal 42(1):124-131, DOI 10.2514/1.9036

Yu X, Chang KH, Choi KK (1998) Probabilistic structural durability prediction. AIAA Journal 36(4):628-637, DOI $10.2514 / 2.415$

Zhang XS, de Sturler E, Paulino GH (2017) Stochastic sampling for deterministic structural topology optimization with many load cases: Density-based and ground structure approaches. Computer Methods in Applied Mechanics and Engineering 325:463-487, DOI 10.1016/j.cma.2017.06.035, URL http://dx.doi.org/10.1016/j.cma.2017.06.035

Zhao J, Wang C (2014a) Robust structural topology optimization under random field loading uncertainty. Structural and Multidisciplinary Optimization 50(3):517-522, DOI 10.1007/s00158-014-1119-6

Zhao J, Wang C (2014b) Robust topology optimization under loading uncertainty based on linear elastic theory and orthogonal diagonalization of symmetric matrices. Computer Methods in Applied Mechanics and Engineering 273:204-218, DOI 10.1016/j.cma.2014.01.018, URL http: //dx.doi.org/10.1016/j.cma.2014.01.018

Zhao Q, Chen X, Ma Z, Lin Y (2016) A Comparison of Deterministic, Reliability-Based Topology Optimization under Uncertainties. Acta Mechanica Solida Sinica 29(1):31-45, DOI 10.1016/S0894-9166(16)60005-8

Zheng J, Luo Z, Jiang C, Ni B, Wu J (2018) Non-probabilistic reliability-based topology optimization with multidimensional parallelepiped convex model. Structural and Multidisciplinary Optimization 57(6):2205-2221, DOI 10.1007/ s00158-017-1851-9 